\title{
Identification of the constituents of double-walled carbon nanotubes using Raman spectra taken with different laser-excitation energies
}

Feng Li

Shenyang National Laboratory for Materials Science, Institute of Metal Research, Chinese Academy of Sciences, 72 Wenhua Road, Shenyang 110016, People's Republic of China

S.G. Chou

Department of Chemistry, Massachusetts Institute of Technology, Cambridge, Massachusetts 02139

Wencai Ren

Shenyang National Laboratory for Materials Science, Institute of Metal Research, Chinese Academy of Sciences, 72 Wenhua Road, Shenyang 110016, People's Republic of China

J.A. Gardecki

George R. Harrison Spectroscopy Laboratory, Massachusetts Institute of Technology, Cambridge, Massachusetts 02139

A.K. Swan, M.S. Ünlü, and B.B. Goldberg

Department of Electrical and Computer Engineering, Boston University, Boston, Massachusetts 02215

Hui-Ming Cheng ${ }^{\text {a) }}$

Shenyang National Laboratory for Materials Science, Institute of Metal Research, Chinese Academy of Sciences, 72 Wenhua Road, Shenyang 110016, People's Republic of China

M.S. Dresselhaus

Department of Physics, Massachusetts Institute of Technology, Cambridge, Massachusetts 02139

(Received 28 November 2002; accepted 25 February 2003)

Double-walled carbon nanotubes (DWNTs), synthesized by the catalytic decomposition of methane, were explored by resonance Raman spectroscopy using different energies for laser excitation. Based on the radial breathing mode frequencies, the indices of the two layers of a DWNT were approximately assigned, depending on the interlayer separation of the two coaxial layers of the DWNT, which ranged from 0.34 to $0.40 \mathrm{~nm}$. From the tentatively assigned results, it was found that the two walls of the DWNT are not strongly selected by chirality and diameter. The results, however, suggest that, for the tubes that are resonant with the available laser excitation energies, most of the outer layers of the observed DWNTs in our samples are semiconducting, while the inner layers of the observed DWNTs are either semiconducting or metallic based on the assembled DWNTs. The characteristics of the G, D, and $\mathrm{G}^{\prime}$ band of the DWNTs are discussed, and a double peak feature in the $\mathrm{D}$ and $\mathrm{G}^{\prime}$ band, originating from the inner and outer layers of the DWNTs, is reported.

\section{INTRODUCTION}

In general, carbon nanotubes can be classified into multi-walled carbon nanotubes (MWNTs) and singlewalled carbon nanotubes (SWNTs). Double-walled carbon nanotubes (DWNTs), which can be considered as two coaxial SWNTs, are a limiting case between SWNTs and MWNTs, and are very important from both a theoretical and experimental standpoint. ${ }^{1}$ SWNTs can be either semiconducting $(\mathrm{S})$ or metallic $(\mathrm{M})$ depending on

a Address all correspondence to this author. e-mail: cheng@imr.ac.cn their diameters and chiralities. Therefore, DWNTs can be divided into four I-O types: S-S, S-M, M-S, and M-M, where the sequence I-O refers to the inner and outer layers, respectively. Thus, an S-M or M-S DWNT can be, respectively, a molecular conductive wire covered by an insulator or a molecular capacitor in a memory device. ${ }^{1}$ Understanding the growth mechanism of DWNTs might help us find a way to control the growth process to obtain the desired DWNT for device applications.

Recently, DWNTs have received much attention in both synthesis and properties research. Hutchison et al. synthesized DWNTs by a hydrogen arc discharge method, ${ }^{2}$ and S. Bandow et al. and Luzzi et al. synthesized DWNTs through the coalescence of $\mathrm{C}_{60}$ in SWNTs 
at high temperature. ${ }^{3,4}$ Moreover, several research groups obtained large quantities of DWNTs by the catalytic decomposition of methane, while the chemical vapor deposition (CVD) process is used to control the reaction parameters precisely. ${ }^{5-7}$ However, the two constituent tubes of the DWNTs have not yet been characterized in detail. Basca et al. ${ }^{8}$ and Bandow et al. ${ }^{3,9}$ characterized their DWNTs by measuring the Raman spectra, and most of their radial breathing mode (RBM) frequencies $\omega_{\mathrm{RBM}}$ were found to be greater than $200 \mathrm{~cm}^{-1}$, which are in accordance with DWNTs that have small diameter inner constituents. They have also found the Raman signal to be weak, which is probably caused by the low concentration of DWNTs in their samples. At the same time, since not all the tubes in the samples were in resonance with the single laser excitation energy used, it is expected that some of the DWNT constituents in the sample were overlooked.

In a previous work, ${ }^{5}$ we reported results on DWNTs prepared by a CVD method, using the catalytic decomposition of methane in the presence of $\mathrm{Fe}$ catalyst particles at $1373 \mathrm{~K}$. Under optimal preparation conditions, the prepared samples are more than 70\% DWNTs, based on high-resolution transmission electron microscopy (HRTEM) observation. The remaining constituents of the samples are mostly catalyst particles encapsulated in graphite layers and amorphous carbon. A few SWNTs and three-walled carbon nanotubes are occasionally found. The outer and inner tube diameters of the DWNTs, as determined from HRTEM images, are mostly in the range of $1.6-2.9 \mathrm{~nm}$ and $0.8-2.0 \mathrm{~nm}$, respectively, having a mean value of 1.52 and $2.26 \mathrm{~nm}$, as obtained from a Gaussian distribution analysis. Moreover, the interlayer spacing of DWNTs is not a constant, ranging from $0.34 \mathrm{~nm}$ to $0.41 \mathrm{~nm} .^{5}$ In this paper, our DWNT samples were characterized by their Raman spectra using four different laser excitation energies, which are in resonance with many of the inner and outer tubes present in the sample. Furthermore, the Raman spectra of the two constituents of the DWNTs are analyzed, based on their RBM frequencies $\omega_{\mathrm{RBM}}$.

\section{EXPERIMENTAL}

DWNTs were prepared by the catalytic decomposition of methane, as detailed in Ref. 4. In our experiment, methane was used as the carbon source, hydrogen as the carrier gas, and ferrocene as the catalyst precursor. During the DWNT preparation, methane, ferrocene, and thiophene vapors were mixed and carried into the reactor, which was maintained at $1373 \mathrm{~K}$. Under optimal preparation conditions, relatively pure DWNTs were obtained. ${ }^{5}$ The bulk morphology of the DWNTs was weblike, which looked semitransparent and light in color.
Raman spectra from the DWNTs were obtained using three Raman systems: (i) a micro-Raman spectrometer (Renishaw 1000B, United Kingdom, objective $\times 50$ ) equipped with an air cooled charge-coupled device and notch filters, (ii) a micro-Raman spectrometer (Jobin Yvon HR800, France, objective $\times 50$ ), and (iii) a modular research micro-Raman spectrograph with Kaiser optical system (Hololab 5000R). The $488.0 \mathrm{~nm}(2.54 \mathrm{eV})$ and $514.5 \mathrm{~nm}(2.41 \mathrm{eV})$ lines from an $\mathrm{Ar}^{+}$laser in system (i), the $632.8 \mathrm{~nm}(1.96 \mathrm{eV})$ line from a He-Ne laser in system (ii), and the $785 \mathrm{~nm}(1.58 \mathrm{eV})$ line from a Ti: sapphire laser in system (iii) were used to obtain the Raman spectra. The laser power impinging on the samples was controlled to be less than $1 \mathrm{~mW}$ over laser spot of approximately $1 \mu \mathrm{m}^{2}$.

\section{RESULTS AND DISCUSSION}

The resonance Raman spectra of SWNTs show two main features: the RBM band and the G band, with the RBM frequency $\omega_{\text {RBM }}$ providing information about the SWNT diameter. Under special circumstances, the index numbers $(n, m)$ can be obtained. In the 1250 $1400 \mathrm{~cm}^{-1}$ range, the disordered-induced D band, which arises from the double resonance Raman scattering process of non-zone-center phonons, can also be observed. The structure of a DWNT consists of two coaxial SWNTs. Theoretical calculations have indicated that the interlayer interaction does not open an energy gap in DWNTs with diameters greater than $0.9 \mathrm{~nm}$. ${ }^{1}$ Thus, as a first approximation, the outer and inner layers retain the basic electronic properties of each constituent graphene monolayer tube. ${ }^{1}$ We assume that the effects of the vibrational and electronic states coupling between adjacent graphene layers are not of sufficient strength to significantly affect the physical properties of the constituent SWNTs. ${ }^{1}$

In terms of intertube interactions, the weak interaction between the outer layers of the DWNTs is similar to the intertube coupling within a SWNT bundle because the intertube separations are comparable in the two cases. However, the number of DWNTs in an average DWNT bundle in our sample is smaller than the number of tubes in a SWNT bundle. ${ }^{5}$ In addition, the relatively small line widths of the Raman features associated with the inner tubes indicate that this interaction is weak, and the transitions are free from the band broadening effects induced by intertube interactions. Since this interaction is weak, we neglect it as a first approximation, and consider the spectral intensities to be dominated by the selection rules for the two isolated SWNTs constituting the DWNT.

The RBMs of DWNTs, associated with both the inner tubes and outer tubes, can each be observed in the resonance Raman spectra. It is assumed that a frequency upshift should be observed due to the interactions between the outer and inner layers of the DWNT. A 
typical Raman spectrum for our DWNT is shown in Fig. 1(a). The resonance Raman spectra of these DWNTs have features that are similar to that of SWNTs: $:^{10}$ the RBMs, a D band, a G band, some higher-order features such as a strong $\mathrm{G}^{\prime}$ band, and an overtone band near approximately $3200 \mathrm{~cm}^{-1}(2 \mathrm{G})$.

The special features of the RBM spectra are shown in Figs. 1(b), 1(c), 1(d), and 1(e) for those DWNTs that are resonant with the various incident $E_{\text {laser }}$ values. In Figs. 1(b)-1(d), the RBM frequencies can be divided into two well-delineated groups associated with the inner and outer layers of the DWNTs. In Fig. 1(c), for example, the groups centered at 130 and $200 \mathrm{~cm}^{-1}$, respectively, are tentatively identified as the low frequency and high frequency RBMs originating from the outer and inner SWNT constituents of the DWNTs. This implies that $E_{22} \mathrm{~s}$ and $E_{11}^{\mathrm{M}}$ satisfy the resonance condition for the inner layers, while $E_{33} \mathrm{~S}$ and $E_{44} \mathrm{~S}$ satisfy the resonance condition for the outer layers. This suggests that the inner and outer layer pairs, made by pairing up tentatively assigned DWMTs, satisfy the $\left[E_{22}{ }^{\mathrm{S}}\right.$ and $\left.E_{33}{ }^{\mathrm{S}}\right]$ or $\left[E_{11}{ }^{\mathrm{M}}\right.$ and $\left.E_{44}{ }^{\mathrm{S}}\right]$ resonance conditions, respectively. The diameter distributions for the inner and outer constituents of the DWNTs were determined by HRTEM measurements on representative DWNT samples taken from the batches used in the Raman experiments, and the diameter distributions obtained by TEM were consistent with the ones determined from the Raman spectrum shown in Fig. 1(c).

However, the $\omega_{\text {RBM }}$ grouping shown in Figs. 1(b) and 1(d) appears to be different from Fig. 1(c), and the groups at low frequency are downshifted. Since a resonanceenhanced Raman signal from many large diameter nanotubes could not be detected, the RBM bands could not be assigned to the inner or outer layers clearly. Also, in

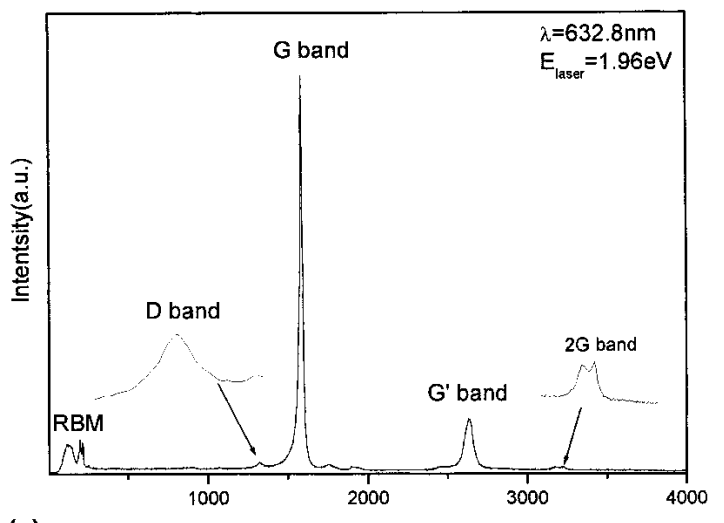

(a)

Raman Shift(cm-1)
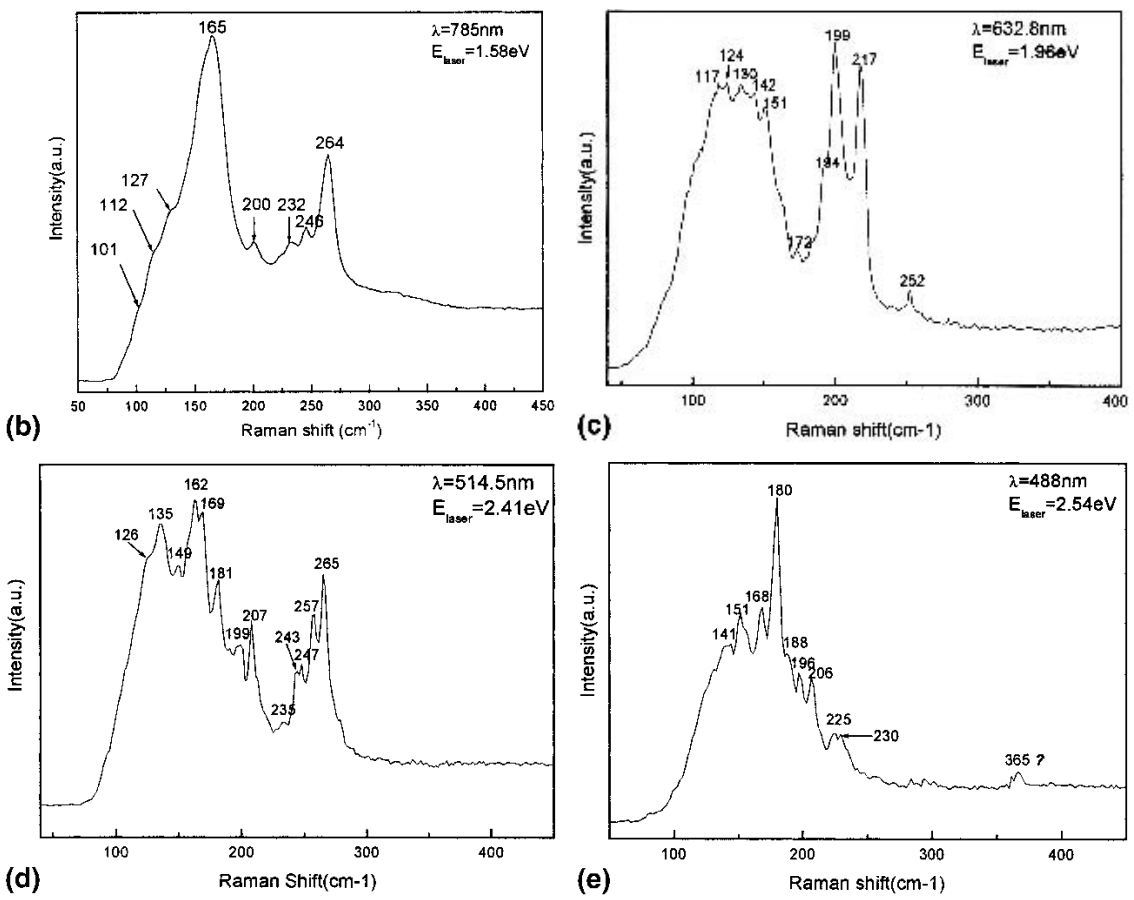

FIG. 1. (a) Typical Raman spectrum of DWNTs excited by $1.96 \mathrm{eV}$ laser, and RBM spectra of DWNTs with different $\mathrm{E}_{\text {laser }}$ values of (b) $2.54 \mathrm{eV}$, (c) $2.41 \mathrm{eV}$, (d) $1.96 \mathrm{eV}$, and (e) $1.58 \mathrm{eV}$. The Lorentz fitting process was used to obtain the positions of the RBM frequencies. 
Fig. 1(e), the RBM spectrum does not clearly divide into two groups because a large number of tubes with different diameters are in resonance with the same $E_{\text {laser }}$ excitation. The line shape of the RBM spectra in Figs. 1(b)-1(e) is broad, indicating that different groups of inner and outer walls of the nanotubes in our sample are resonant with each $E_{\text {laser. }}$. The intensity of the RBM band is much larger, and the spectra are more complex than those in previously reported works on DWNTs. ${ }^{3,7}$ The complexity probably arises from the mixing of many different $\left(n, m ; n^{\prime}, m^{\prime}\right)$ constituents in our DWNT samples, where the pairs of integers in brackets denote the inner $(n, m)$ and outer tubes $\left(n^{\prime}, m^{\prime}\right)$, respectively.

The $\omega_{\mathrm{RBM}}$ of a carbon nanotube is inversely proportional to its diameter because the resonance condition is very sensitive to the nanotube structure. This makes it possible for us to determine the diameter distribution by using different laser excitation energies to cover a representative sampling of inner and outer constituents of the DWNTs. ${ }^{10,11}$ The nanotube diameter $d_{\mathrm{t}}$ for the inner and outer constituents is obtained using the relation $\left(d=248 / \omega_{\mathrm{RBM}}\right)$, which was established by making Raman measurements on individual SWNTs sitting on a $\mathrm{Si} / \mathrm{SiO}_{2}$ substrate. ${ }^{11}$ From our DWNT measurements, we observed resonant nanotube signals that correspond to diameters ranging from 0.67 to $2.5 \mathrm{~nm}$ (including inner and outer diameters) in our samples, using the 488 , 514.5, 632.8, and $785 \mathrm{~nm}$ laser lines.

Subsequently, the spectra in Figs. 1(b)-1(e) are tentatively assigned $(n, m)$ indices, based on their $d_{\mathrm{t}}$ and $E_{\mathrm{ii}}$ values, ${ }^{10}$ except for the two modes observed in Fig. 1(b) at $200 \mathrm{~cm}^{-1}$ and Fig. 1(c) at $252 \mathrm{~cm}^{-1}$ that could not be assigned, based on tight-binding calculations. Nanotubes with the same $\omega_{\text {RBM }}$ that are resonant with different laser excitation energies, such as $168 \mathrm{~cm}^{-1}$ in Fig. 1(e) and $169 \mathrm{~cm}^{-1}$ in Fig. 1(d), are assigned the same $(n, m)$ indices. On the other hand, within the resonance window of a given excitation energy, more than one set of $(n, m)$ index can be assigned to a given $\omega_{\mathrm{RBM}}$. For example, $\omega_{\mathrm{RBM}}=246 \mathrm{~cm}^{-1}$ in Fig. 1(b) can be assigned to $(8,7)$ or $(11,3)$ since they both are within the resonant windows of the $1.58 \mathrm{eV}$ laser excitation. The differences between the experimental $\omega_{\mathrm{RBM}}$ and the theoretical assignments for these $d_{\mathrm{t}}$ values are less than $4 \%$. The results from this analysis are shown in Table I. The tentative results listed in Table I indicate that all chiralities for inner and outer tubes could be found, and it seems that DWNTs do not prefer any special chiralities for their constituent layers.

Based on a tight binding calculation $\left(\gamma_{0}=2.90 \mathrm{eV}\right.$, $\left.a_{\mathrm{c}-\mathrm{c}}=0.142 \mathrm{~nm}\right)$ for SWNTs, the tubes with $0.6 \leqslant \mathrm{~d}_{\mathrm{t}}$ $\leqslant 3.0 \mathrm{~nm}$ and with resonant windows $( \pm 0.1 \mathrm{eV})$ within the four laser excitation energies $\left(E_{\text {laser }}=1.58\right.$, $1.96,2.41$, and $2.54 \mathrm{eV}$ ), are shown by the shaded region in Fig. 2. Presently, there are no corresponding calculations available for DWNTs. In this sense, the results of Fig. 2 for SWNTs should be considered as approximations for DWNTs. As stated above, the intertube interactions are more important for the outer tubes of the DWNTs, whereas the curvature effects are more important for the inner tubes. Both interactions give rise to corrections to the $E_{\mathrm{ii}}$ values given in Fig. 2. Previous theoretical calculations have predicted that small diameter tubes $\left(d_{\mathrm{t}}<0.9 \mathrm{~nm}\right)$ do not always follow the tight binding calculation. ${ }^{10}$ Since most of the diameter determinations based on the RBM frequency in Raman experiments use tight binding results for their energies, departure from tight binding predictions can lead to deviations in diameter and $(n, m)$ assignments for small diameter tubes. ${ }^{12}$

In Fig. 2, the tubes that are resonant with $E_{\text {laser }}$ below $1.53 \mathrm{eV}$, between 1.68 and $1.91 \mathrm{eV}$, from 2.01 to $2.36 \mathrm{eV}$, and above $2.59 \mathrm{eV}$ cannot be observed with our four available laser lines. Also, as a result of the notch filter cut-off, we cannot measure Raman shifts less than $100 \mathrm{~cm}^{-1}\left(d_{\mathrm{t}}>2.5 \mathrm{~nm}\right)$. We can therefore see that some tube diameters, which can form DWNTs, are not in resonance with any of the four laser excitation energies. Furthermore, tubes with large diameters $\left(d_{\mathrm{t}}>3.0 \mathrm{~nm}\right)$ exhibit only a small resonant enhancement effect, and therefore, it is more difficult to detect their RBM. Since the outer and inner tube diameters of the DWNTs, from HRTEM images, are approximately in the ranges of $1.6-3.6 \mathrm{~nm}$ and $0.8-2.8 \mathrm{~nm}$, respectively, ${ }^{5}$ we concluded that the diameter distribution of the tubes probed by the resonance Raman experiment is different from that obtained from HRTEM results.

Theoretical calculations indicate that the stability of a DWNT depends only on the interlayer spacing, which reaches an energy minimum when the mean interlayer separation equals $0.34 \mathrm{~nm}$, independent of the chiralities of the two constituent tubes. ${ }^{13}$ The most important parameter is then the distance between the two layers of the DWNTs based on the indices of the tubes. Previous experimental results have shown that the average interlayer distance of MWNTs increases with the number of layers in a MWNT. ${ }^{14}$ The interlayer distance of the large diameter MWNTs $(>10 \mathrm{~nm})$ is close to that of single-crystal graphite $(0.335 \mathrm{~nm})$. In our case, the experimental results suggest that the average interlayer distance of our DWNTs is the largest interlayer distance that could be found in MWNTs. ${ }^{14}$ Basca et al. ${ }^{8}$ has reported the interlayer distance for DWNTs of small diameters to be smaller than that of graphite, but such small interlayer separations were not observed in our results. In general, it is not expected that DWNTs will have an interlayer space smaller than that for crystalline graphite. We have found the interlayer separation for our sample to be closer to those reported for the DWNTs synthesized through hightemperature annealing $\left(1200{ }^{\circ} \mathrm{C}\right)$ of SWNTs filled with 
F. Li et al.: Identification of the constituents of DWNTs using Raman spectra taken with different laser-excitation energies

TABLE I. RBM frequencies, diameters, conductivity, assigned indices, diameters calculated from assigned indices and the assembled DWNTs.

\begin{tabular}{|c|c|c|c|c|c|c|c|c|c|c|}
\hline$E_{\text {laser }}$ & 785 & 785 & 632.8 & 632.8 & 514.5 & 785 & 632.8 & 514.5 & 632.8 & 488 \\
\hline$\omega_{\mathrm{RBM}}$ & 101 & 112 & 117 & 124 & 126 & 127 & 130 & 135 & 142 & 141 \\
\hline$d_{t}$ & 2.46 & 2.21 & 2.12 & 2.00 & 1.97 & 1.95 & 1.92 & 1.84 & 1.75 & 1.76 \\
\hline Cond $^{\mathrm{a}}$ & $\mathrm{S}$ & $S$ & $\mathrm{~S}$ & $S$ & $\mathrm{M}$ & $\mathrm{S}$ & $S$ & $S$ & $\mathrm{~S}$ & $S$ \\
\hline $\mathrm{n}, \mathrm{m}$ & $(26,9)$ & $(24,7)$ & $(19,12)$ & $(20,9)$ & $(16,13)$ & $(19,9)$ & $(24,1)$ & $(23,1)$ & $(18,7)$ & $(20,4)$ \\
\hline $\mathrm{d}_{\mathrm{t}} \mathrm{Ca}^{\mathrm{b}}$ & 2.46 & 2.20 & 2.12 & 2.01 & 1.97 & 1.94 & 1.92 & 1.84 & 1.75 & 1.74 \\
\hline
\end{tabular}

TABLE I. RBM frequencies, diameters, conductivity, assigned indices, diameters calculated from assigned indices and the assembled DWNTs. (continued)

\begin{tabular}{lcccccccccc}
\hline \hline $\mathrm{E}_{\text {laser }}$ & 488 & 632.8 & 488 & 5145 & 632.8 & 785 & 514.5 & 488 & 632.8 & 785 \\
$\omega_{\text {RBM }}$ & 188 & 194 & 196 & 199 & 199 & 200 & 207 & 206 & 217 \\
$\mathrm{~d}_{\mathrm{t}}$ & 1.32 & 1.28 & 1.27 & 1.25 & 1.25 & 1.24 & 1.20 & 1.20 & 1.14 \\
$\mathrm{C}$ Cond & $\mathrm{S}$ & $\mathrm{M}$ & $\mathrm{S}$ & $\mathrm{S}$ & $\mathrm{M}$ & $\cdots$ & $\mathrm{S}$ & $\mathrm{S}$ & $\mathrm{M}$ \\
$\mathrm{n}, \mathrm{m}$ & $(12,7)$ & $(11,8)$ & $(10,9)$ & $(16,0)$ & $(12,6)$ & $\cdots$ & $(11,7)$ & $(12,5)$ & $(10,7)$ & $(11,4)$ \\
$\mathrm{d}_{\mathrm{t}} \mathrm{Ca}$ & 1.30 & 1.29 & 1.29 & 1.25 & 1.24 & $\cdots$ & 1.23 & 1.18 & 1.16 & 1.05 \\
\hline
\end{tabular}

TABLE I. RBM frequencies, diameters, conductivity, assigned indices, diameters calculated from assigned indices and the assembled DWNTs. (continued)

\begin{tabular}{|c|c|c|c|c|c|c|c|c|c|c|}
\hline $\mathrm{E}_{\text {laser }}$ & 514.5 & 632.8 & 488 & 514.5 & 785 & 488 & 514.5 & 632.8 & 488 & 514.5 \\
\hline$\omega_{\mathrm{RBM}}$ & 149 & 151 & 151 & 162 & 165 & 168 & 169 & 172 & 180 & 181 \\
\hline$d_{t}$ & 1.66 & 1.64 & 1.64 & 1.53 & 1.50 & 1.48 & 1.47 & 1.44 & 1.38 & 1.37 \\
\hline Cond & S & $\mathrm{S}$ & S & S & M & $\mathrm{S}$ & S & M & $\mathrm{S}$ & $\mathrm{S}$ \\
\hline $\mathrm{n}, \mathrm{m}$ & $(14,10)$ & $(18,5)$ & $(18,5)$ & $(15,7)$ & $(14,8)$ & $(17,3)$ & $(17,3)$ & $(17,2)$ & $(15,4)$ & $(11,9)$ \\
\hline $\mathrm{d}_{\mathrm{t}} \mathrm{Ca}$ & 1.63 & 1.64 & 1.64 & 1.52 & 1.51 & 1.46 & 1.46 & 1.42 & 1.36 & 1.36 \\
\hline
\end{tabular}

TABLE I. RBM frequencies, diameters, conductivity, assigned indices, diameters calculated from assigned indices and the assembled DWNTs. (continued)

\begin{tabular}{|c|c|c|c|c|c|c|c|c|c|c|}
\hline$E_{\text {laser }}$ & 488 & 5145 & 785 & 785 & 514.5 & 632.8 & 514.5 & 785 & 514.5 & 488 \\
\hline$\omega_{\mathrm{RBM}}$ & 230 & 235 & 246 & 246 & 243,247 & 252 & 257 & 264 & 265 & 365 \\
\hline$d_{t}$ & 1.08 & 1.06 & 1.01 & 1.01 & 1.00 & 0.98 & 0.96 & 0.94 & 0.94 & 0.68 \\
\hline Cond $^{\mathrm{a}}$ & M & M & $S$ & $\mathrm{~S}$ & M & $\ldots$ & $\mathrm{M}$ & $\mathrm{S}$ & $\mathrm{M}$ & $\mathrm{S}$ \\
\hline $\mathrm{n}, \mathrm{m}$ & $(13,1)$ & $(13,1)$ & $(8,7)$ & $(11,3)$ & $(9,6)$ & $\ldots$ & $(10,4)$ & $(12,1)$ & $(11,2)$ & $(8,1)$ \\
\hline $\mathrm{d}_{\mathrm{t}} \mathrm{Ca}^{\mathrm{b}}$ & 1.06 & 1.06 & 1.02 & 1.00 & 1.02 & $\ldots$ & 0.98 & 0.98 & 0.95 & 0.67 \\
\hline
\end{tabular}

DWNT $_{\text {S-S }} \quad(8,1 ; 11,9)(8,1 ; 15,4)(8,1 ; 17,3)(12,1 ; 18,7)(12,1 ; 20,4)(8,7 ; 18,7)(8,7 ; 20,4)(8,7 ; 21,3)(11,3 ; 20,4)(11,3 ; 18,7)(11$, $4 ; 18,7)(11,4 ; 20,4)(11,4 ; 23,1)(12,5 ; 24,1)(12,5 ; 19,9)(11,7 ; 24,1)(11,7 ; 19,9)(11,7,20,9)(16,0 ; 19,9)(16,0 ; 20,9)$ $(10,9 ; 20,9)(10,9 ; 19,12)(10,9 ; 19,12)(12,7,20,9)(11,9 ; 19,12)(15,4 ; 19,12)(17,3 ; 24,7)(15,7 ; 24,7)(18,5 ; 26,9)(14$, $10 ; 26,9)(18,7 ; 26,9)(23,1,26,9)$

DWNT $_{\mathrm{M}-\mathrm{S}}(11,2 ; 18,5)(11,2 ; 14,10)(11,2 ; 18,7)(11,2 ; 20,4)(10,4 ; 18,7)(10,4 ; 20,4)(9,6 ; 18,7)(9,6 ; 18,7)(9,6 ; 20,4)(13,1 ; 18,7)$ $(13,1 ; 20,4)(13,1,23,1)(10,7 ; 23,1)(10,7 ; 23,1)(10,7 ; 24,1)(10,7 ; 19,9)(12,6 ; 24,1)(12,6 ; 19,9)(12,6 ; 20,9)(11,8 ;$ $20,9)(17,2 ; 19,12)(14,8 ; 24,7)$

DWNT $_{\text {S-M }} \quad(8,1 ; 17,2)(11,7 ; 16,13)(16,0 ; 16,13)(10,9 ; 16,13)(12,7 ; 16,13)$

DWNT $_{M-M}(12,5 ; 16,13)(12,6 ; 16,13)(11,8 ; 16,3)$

${ }^{a}$ Cond. means the conductive type of the tubes.

${ }^{b} d_{t}$ Ca. means the diameters obtained from the index (n, m)

DWNT can be assembled by any tubes based on distance of layers from 0.34 to $0.40 \mathrm{~nm}$.

$\mathrm{C}_{60}$ when the Raman spectra of the two are compared. ${ }^{3}$ The interlayer separations of these pea-pod derived DWNTs were found to range from 0.335 to $0.37 \mathrm{~nm},{ }^{3}$ and recently reported results give the value of approximately $0.35 \mathrm{~nm} .{ }^{9}$ As the HRTEM studies indicated, the interlayer spacing of the DWNT is not a constant, ranging from 0.335 to $0.41 \mathrm{~nm} .{ }^{5}$ Therefore, an interlayer spacing in the range of 0.335 to $0.40 \mathrm{~nm}$ was taken into consideration when we assembled our DWNT pairs.
The indices of possible inner and outer layer tubes that can be used to assemble DWNTs are shown in Table I. For the assembled DWNTs, the inner and outer constituent SWNTs can either be identified by the same $E_{\text {laser }}$ excitation, such as $(10,7 ; 24,1)$, where both constituents are in resonance with the $632.8 \mathrm{~nm}$ laser excitation, or by being resonant with different laser lines. As an example, for the $(12,5 ; 24,1)$ tube, the inner and outer tubes are in resonance with the 488 and $632.8 \mathrm{~nm}$ lines, 


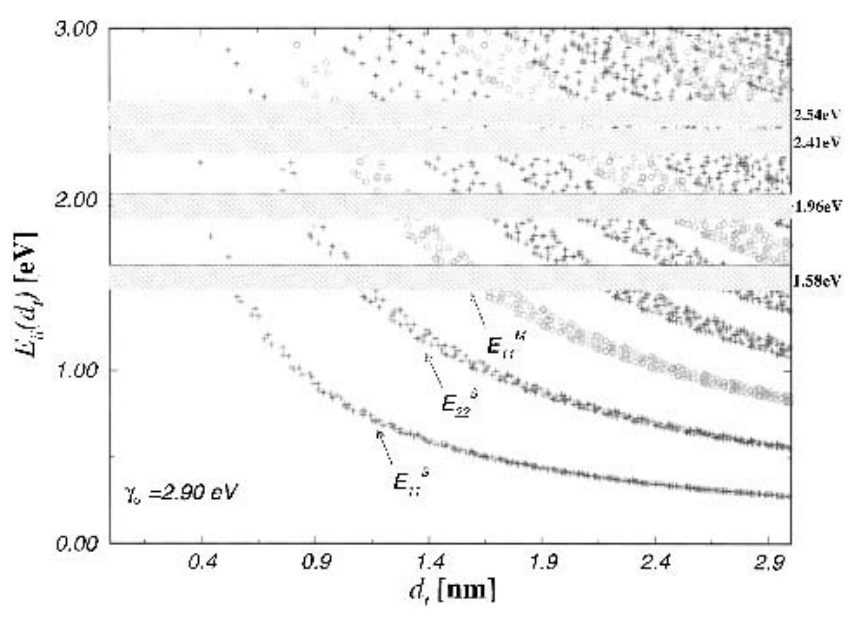

FIG. 2. Relationship between the tube that is resonant with each $E_{\text {laser }}$ based on the dependence of each electronic transition $E_{\mathrm{ii}}$ on tube diameter between $0.6<d_{t}<3.0 \mathrm{~nm}$ predicted from tight-binding calculations. $^{8}$

respectively. Most of the diameters of the assembled DWNTs can be confirmed by HRTEM observation except for the small-diameter DWNTs, such as $(8,1 ; 11,9)$, $(8,1 ; 15,4)$, and $(8,1 ; 17,3)$. From Table I, we learn that two DWNTs with the same outer diameters could have different diameter inner tubes. This observation is confirmed by our HRTEM measurements. ${ }^{5}$ The tentatively assembled DWNTs indicate that all combinations of diameters and chiralities are possible for the two layers of nanotubes, provided that the minimum interlayer separation is maintained.

Since large-diameter nanotubes were not detected in the RBM Raman spectra and the DWNT bundles caused each $\omega_{\text {RBM }}$ to shift by a small amount due to the intertube interactions, the average diameter of the inner and outer constituents of the DWNTs, as inferred from the Raman measurements, are somewhat smaller than the HRTEM observation. As mentioned before, many of the tubes are outside of the resonant windows. Since they could not be observed, in the Raman spectra they are not considered in forming the DWNT pairs listed in Table I. Thus, the Raman experiments given here do not provide a quantitative determination of the diameter distribution in the sample. Instead, Table I provides a guide on how a detailed structure determination could be carried out.

If the nanotube growth process is random, then onethird of the tubes would be expected to be metallic, and two-thirds are semiconducting. ${ }^{10}$ This implies that the ratio of S-S:M-S:S-M:M-M should be 4:2:2:1. However, the assembled DWNT, include $32 \mathrm{~S}-\mathrm{S}, 20 \mathrm{M}-\mathrm{S}, 5 \mathrm{~S}-\mathrm{M}$, and 3 M-M DWNTs, as shown in Table I. The ratio of S-S and M-S or S-M and M-M is close to two. But the ratio of M-S to S-M pairs is far from one. This suggests, that for the diameter distribution of this sample and the available laser excitation energies, the outer layers of the DWNTs are predominately semiconducting.
Whether the experiments favor either metallic or semiconducting tubes depends on the resonance condition, and the probability of finding a given tube is strongly dependent on the laser lines that were used.

During the CVD process, the outer shells of the DWNTs form first, and then the inner shell forms by transferring carbon atoms between the inner and outer layers through a lip-lip interaction ${ }^{14}$ with the number of carbon atoms increasing to satisfy the minimum energy requirement. From the above analysis, since the DWNTs in our samples are structurally mixed, with different diameter pairs, and since the Raman spectra can only identify constituents one at a time, it is very difficult to be sure how the inner and outer tubes assemble to form DWNTs in bundle samples. However, if isolated DWNTs or very dilute samples were obtained, we could perhaps find the two constituents of DWNTs directly from the RBM frequency and use the information from the $G$ band and other features to corroborate the tentative assignments, as is done when doing $(n, m)$ assignments for the isolated SWNTs. ${ }^{15}$ From the above discussions, we can see that the assembled DWNTs depend on the choice of the energy overlap integral $\gamma_{0}$. We found that the value of $\gamma_{0}=2.90 \mathrm{eV}$ provided the best fit to our experimental results for isolated SWNTs. ${ }^{15}$ Although the fits for the assembled DWNTs depend on the choice of the overlap integral, it may be feasible to identify the constituents of DWNTs from the energy overlap integrals appropriately modeled in further experimental studies and theoretical calculations. However, the determination of a modified energy overlap integral for DWNTs is beyond the scope of the present paper.

We use the resonantly enhanced $G$ band features of the DWNTs to distinguish between resonant metallic and semiconducting tubes according to their inner or outer layers. As for the results from isolated

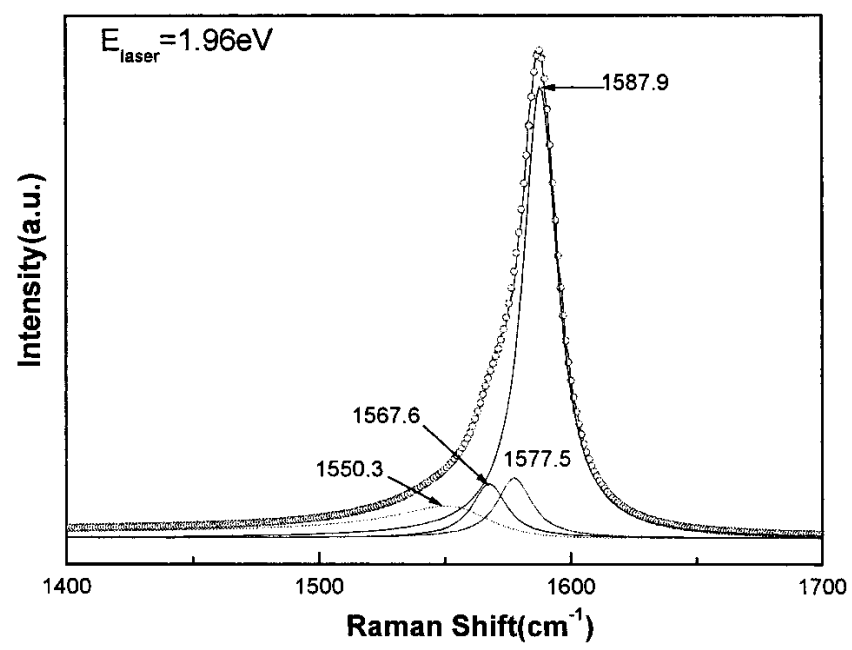

FIG. 3. G band and fitting result of the DWNTs with $632.8 \mathrm{~nm}$ laser excitation. 
SWNTs, ${ }^{15}$ the $\mathrm{G}$ band of a carbon nanotube consists of two peaks, $\mathrm{G}^{+}$(with $\omega_{\mathrm{G}+} \sim 1590 \mathrm{~cm}^{-1}$ ) and $\mathrm{G}^{-}$, which are associated with the motion of carbon-atom displacements along the tube axis and the circumferential directions, respectively. Figure 3 gives the $\mathrm{G}^{-}$band Raman spectra of DWNTs using $632.8 \mathrm{~nm}$ laser excitation. From the RBM analysis, we conclude that the Raman G band features for the incident photons are attributed to the inner layer metallic or semiconducting tubes, when they are respectively resonant with the $E_{11}{ }^{\mathrm{M}}$ or $E_{22}{ }^{\mathrm{S}}$ van Hove singularities in the joint density of states, and $E_{33} \mathrm{~S}$ or $E_{44} \mathrm{~S}$ satisfies the resonance condition for the tubes in the outer layers. The modes in Fig. 3 are identified as four modes: one $\mathrm{G}^{+}$mode and three $\mathrm{G}^{-}$modes. Out of the three $\mathrm{G}^{-}$modes, one is for a metallic tube with a BreitWigner-Fano line shape, ${ }^{16}$ in accordance with an inner tube satisfying the $E_{11}{ }^{\mathrm{M}}$ resonance condition, and two are for semiconducting tubes with Lorentzian line shapes, in accordance with the inner and outer layers $\left(E_{22} \mathrm{~s}\right.$ or $E_{33} \mathrm{~s}$ or $E_{44} \mathrm{~S}$ ). Experiments at the single SWNT level show that the splitting between $\mathrm{G}^{+}$and $\mathrm{G}^{-}$obeys the diameter dependence $^{15}$ characterized by Eq. (1):

$$
\omega_{\mathrm{G}-}=\omega_{\mathrm{G}+}-\frac{C}{d_{\mathrm{t}}^{2}},
$$

where $C=47.7 \mathrm{~cm}^{-1} \mathrm{~nm}^{2}$ for semiconducting tubes and $79.5 \mathrm{~cm}^{-1} \mathrm{~nm}^{2}$ for metallic nanotubes.

Based on Eq. (1), the diameters of the metallic inner, the semiconducting inner, and the outer tubes of the DWNTs can be evaluated, yielding 1.45, 1.53, and $2.14 \mathrm{~nm}$, respectively, from the data presented in Fig. 3. They are just within the excitation windows (Fig. 2) and close to the diameters obtained from the HRTEM images. We also observe the $\mathrm{G}$ band splitting for all the Raman spectra with the other laser excitation energies.

Most of the DWNTs are formed in bundles due to the Van der Waals attraction between the tubes, and these bundles always consist of many well-aligned DWNTs, which is similar to observations in SWNT bundles. Regarding the D band, the first interesting point in the Raman spectra for the DWNTs is the strong dependence of the D band frequency on $E_{\text {laser }}$, which we find in this work to be similar to that for all $s p^{2}$ carbon materials, independent of their structures. ${ }^{10}$ As the inner and outer layers of the DWNTs have two slightly different resonant conditions due to their structural differences, the $\mathrm{D}$ and $\mathrm{G}^{\prime}$ bands each split into two peaks, which we identify as originating from the inner and outer tubes. The $D$ and $\mathrm{G}^{\prime}$ bands of the DWNTs are shown in Fig. 4. Figure 4(a) shows the D band of the DWNTs with different $E_{\text {laser }}$ excitations. The inset shows the two-peak structure of the DWNTs by $488 \mathrm{~nm}$ laser excitation, originating from the inner and outer layers, respectively, and the peak shifts to higher $\omega_{\mathrm{D}}$ values with increasing $\mathrm{E}_{\text {laser }}$. Figures 4(b) and 4(c) show the linear fit between the laser energy
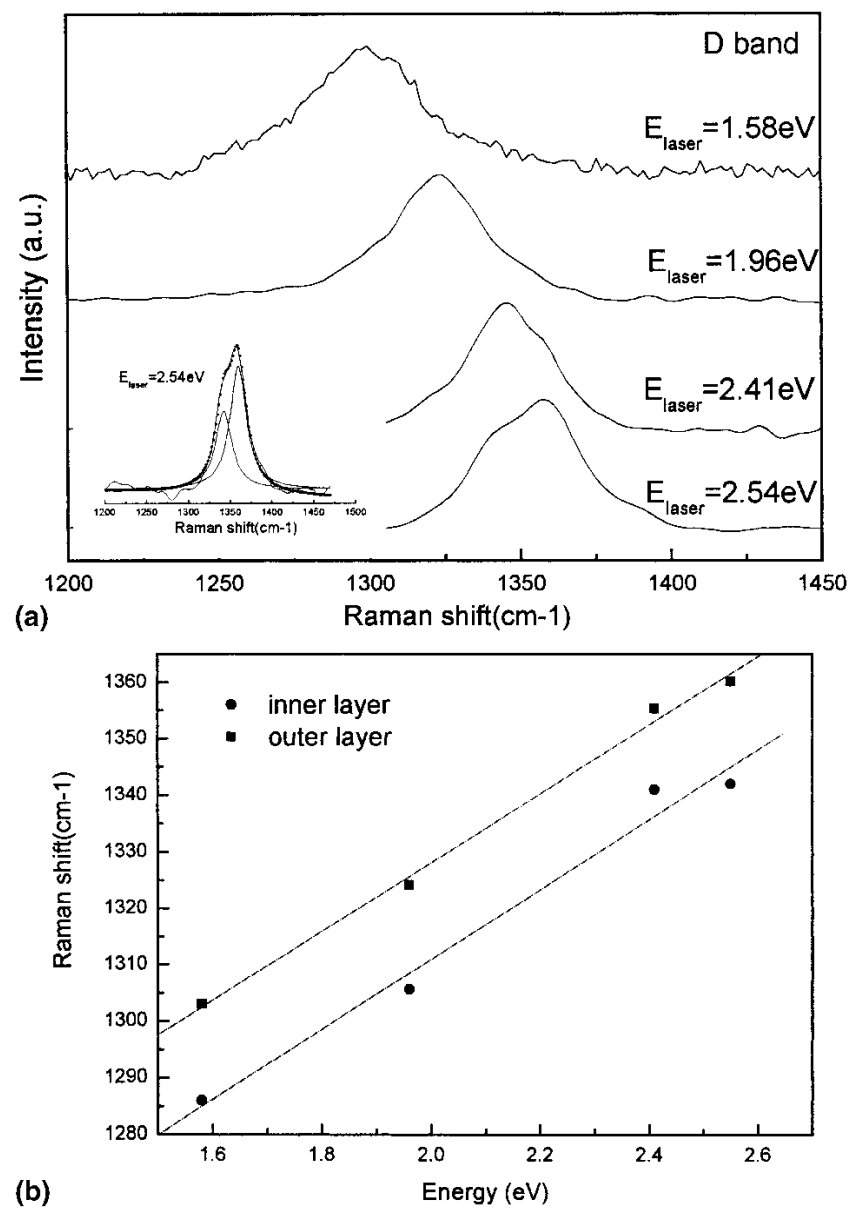

(b)

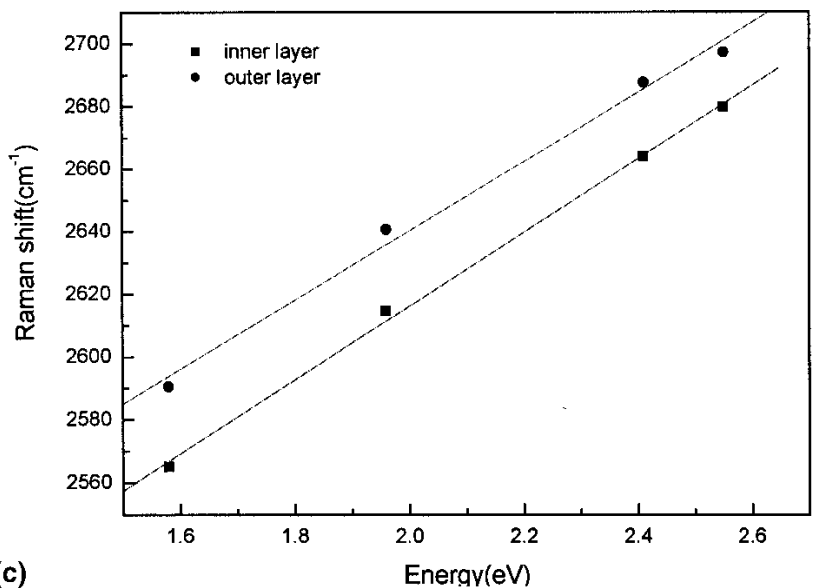

FIG. 4. (a) The relation of the frequency and energy in the D band of the DWNTs; the inset picture shows the splitting structure of the D band with $488 \mathrm{~nm}$ laser excitation. (b) Relation between the Raman frequency and the laser excitation and energy in the D band of the inner and outer layer DWNTs. (c) Relation between the Raman frequency and the laser excitation with the $\mathrm{G}^{\prime}$ band of the inner and outer layers of the DWNTs.

and the Raman shift of the inner- and outer-layer contributions to the D and $\mathrm{G}^{\prime}$ bands in the DWNTs after the peak fitting. The results suggest that the low and high frequencies come from the inner and outer layers of the 
DWNTs since the D and $\mathrm{G}^{\prime}$ band frequencies are proportional to the average $1 / d_{\mathrm{t}}$ of the tubes. ${ }^{15}$ The slope of the inner layers, in Fig 4(b), is essentially parallel with that for the outer layers $\left[\partial \omega_{\text {Din }} / \partial E_{\text {laser }}\left(61.8 \mathrm{~cm}^{-1} / \mathrm{eV}\right) \approx\right.$ $\left.\partial \omega_{\text {Dout }} / \partial E_{\text {laser }}\left(60.9 \mathrm{~cm}^{-1} / \mathrm{eV}\right)\right]$. From Fig. $4(\mathrm{c})$ it was found that the values $\partial \omega_{\mathrm{G}^{\prime} \text { in }} / \partial E_{\text {laser }}\left(117 \mathrm{~cm}^{-1} / \mathrm{eV}\right) \approx$ $\partial \omega_{\text {Gout }} / \partial \mathrm{E}_{\text {laser }}\left(110 \mathrm{~cm}^{-1} / \mathrm{eV}\right)$, are almost twice that of the $\partial \omega_{\text {Din }} / \partial E_{\text {laser }}$ and $\partial \omega_{\text {Dout }} / \partial E_{\text {laser. }}$ The values of these slopes are close to the corresponding values for SWNTs, for which $\partial \omega_{\mathrm{G}^{\prime}} / \partial E_{\text {laser }}=106 \mathrm{~cm}^{-1} / \mathrm{eV}, \partial \omega_{\mathrm{D}} / \partial E_{\text {laser }}=$ $51.2 \mathrm{~cm}^{-1} / \mathrm{eV}$, and are close to the values for graphite, for which $\partial \omega_{\mathrm{G}^{\prime}} / \partial E_{\text {laser }}=101 \mathrm{~cm}^{-1} / \mathrm{eV}, \partial \omega_{\mathrm{D}} / \partial E_{\text {laser }}=$ $48 \mathrm{~cm}^{-1} / \mathrm{eV}$.

\section{CONCLUSIONS}

The indices of the two layers of a DWNT were approximately assigned based on the RBM frequencies, depending on the interlayer separations of the two coaxial layers of the DWNT, which ranged from 0.34 to $0.40 \mathrm{~nm}$. The results suggest that most of the outer layers of the observed DWNTs in our samples are semiconducting, and only a few are metallic, while the inner layers of the observed DWNTs are either semiconducting or metallic based on the assembled DWNTs and the available laser lines. The inner and outer diameters obtained from the $\mathrm{G}$ band are consistent with these predicted by the RBM frequencies. A splitting in the $\mathrm{D}$ band and $\mathrm{G}^{\prime}$ band features in the Raman spectra originating from the inner and outer layers of the DWNTs can also be identified.

\section{ACKNOWLEDGMENTS}

The authors at IMR acknowledge National Natural Science Foundation of China (NSFC) Grant No. 50025204 and CAS for support of this work. The confocal micro-Raman experiment made use of the MRSEC Shared Facilities supported by the NSF under Grant No. DMR-9808941 and NSF Laser Facility Grant No.
0111370-CHE. The project also made use of the MIT Laser Research Facilities of the George R. Harrison Spectroscopy Laboratory. The MIT authors acknowledge financial support under NSF Grant No. DMR 01-16042.

\section{REFERENCES}

1. R. Saito, G. Dresselhaus, and M.S. Dresselhaus, J. Appl. Phys. 73, 494 (1993).

2. J.L. Hutchison, N.A. Kiselev, E.P. Krinichnaya, A.V. Krestinin, R.O. Loutfy, A.P. Morawsky, V.E. Muradyan, E.D. Obraztsova, J. Sloan, S.V. Terekhov, and D.N. Zakharov, Carbon 39, 761 (2001).

3. S. Bandow, M. Takizawa, K. Hirahara, M. Yudasaka, and S. Iijima, Chem. Phys. Lett. 337, 48 (2001).

4. B.W. Smith, M. Monthioux, and D.E. Luzzi, Nature 396, 323 (1998).

5. W.C. Ren, F. Li, J. Chen, S. Bai, and H.M. Cheng, Chem. Phys. Lett. 359, 196 (2002).

6. L. Ci, Z Rao, Z. Zhou, D. Tang, X. Yan, Y. Liang, D. Liu, H. Yuan, W. Zhou, G. Wang, W. Liu, and S. Xie, Chem. Phys. Lett. 359, 63 (2002).

7. R.R. Bacsa, C. Laurent, A. Peigney, W.S. Bacsa, T. Vaugien, and A. Rousset, Chem. Phys. Lett. 323, 566 (2000).

8. R.R. Bacsa, A. Peigney, Ch. Laurent, P. Puech, and W.S. Bacsa, Phys. Rev. B 65, 161404 (2002).

9. S. Bandow, G. Chen, G.U. Sumanasekera, R. Gupta, M. Yudasaka, S. Iijima, and P.C. Eklund, Phys. Rev. B 66, 075416 (2002).

10. M.S. Dresselhaus and P.C. Eklund, Adv. Phys. 49, 705 (2000).

11. A. Jorio, R. Saito, J.H. Hafner, C.M. Lieber, M. Hunter, T. McClure, G. Dresselhaus, and M.S. Dresselhaus, Phys. Rev. Lett. 86, 1118 (2001).

12. A.G. Souza Filho, S.G. Chou, Ge.G. Samsonidze, G. Dresselhaus, M.S. Dresselhaus, L. An, J. Liu, A.K. Swan, M.S. Ünlü, B.B. Goldberg, A. Jorio, and R. Saito (unpublished).

13. R. Saito, R. Matsuo, T. Kimura, G. Dresselhaus, and M.S. Dresselhaus, Chem. Phys. Lett. 348, 187 (2001).

14. C.H. Kiang, M. Endo, P.M. Ajayan, G. Dresselhaus, and M.S. Dresselhaus, Phys. Rev. Lett. 81, 1869 (1998).

15. A. Jorio, A.G. Souza Filho, G. Dresselhaus, M.S. Dresselhaus, A.K. Swan, M.S. Ünlü, B.B. Goldberg, M.A. Pimenta, J.H. Hafner, C.M. Lieber, and R. Saito, Phys. Rev. B. 65, 155412 (2002).

16. S.D.M. Brown, A. Jorio, P. Corio, M.S. Dresselhaus, G. Dresselhaus, R. Saito, and K. Kneipp, Phys. Rev. B. 63, 155414 (2001). 